CERN-TH/2001-229

August, 2001

\title{
Dressed gluon exponentiation
}

\author{
Einan Gardi \\ TH Division, CERN, CH-1211 Geneva 23, Switzerland
}

\begin{abstract}
Perturbative and non-perturbative aspects of differential cross-sections close to a kinematic threshold are studied applying "dressed gluon exponentiation" (DGE). The factorization property of soft and collinear gluon radiation is demonstrated using the light-cone axial gauge: it is shown that the singular part of the squared matrix element for the emission of an off-shell gluon off a nearly on-shell quark is universal. We derive a generalized splitting function that describes the emission probability and show how Sudakov logs emerge from the phase-space boundary where the gluon transverse momentum vanishes. Both soft and collinear logs associated with a single dressed gluon are computed through a single integral over the running-coupling to any logarithmic accuracy. The result then serves as the kernel for exponentiation. The divergence of the perturbative series in the exponent indicates specific non-perturbative corrections. We identify two classes of observables according to whether the radiation is from an initialstate quark, as in the Drell-Yan process, or a final-state quark, forming a jet with a constrained invariant mass, as in fragmentation functions, event-shape variables and deep inelastic structure functions.
\end{abstract}

\footnotetext{
*Research supported in part by the EC program "Training and Mobility of Researchers", Network "QCD and Particle Structure", contract ERBFMRXCT980194.
} 


\section{Introduction}

Many observables in QCD receive large corrections from soft and collinear gluon radiation. Contrary to virtual corrections, real gluon emission has a restricted phase space that depends on the kinematics. In particular, close to a kinematic threshold, a differential cross-section is dictated by soft and collinear gluon radiation. Classical examples are event-shape variables close to the two-jet limit, and fragmentation functions, deep inelastic scattering structure functions and Drell-Yan production for $x \longrightarrow 1$.

Differential cross-sections close to threshold have been studied extensively in perturbation theory, where the phenomenon is reflected in Sudakov $\operatorname{logs} L=\ln 1 /(1-x)$. The presence of large logs in the perturbative coefficients limits the applicability of fixed-order calculations. It is a universally acknowledged conjecture that perturbation theory can be improved by resumming the leading logs to all orders in the coupling [1]-[0].

The resummation of Sudakov logs is based on the general property of factorization. Factorization of soft photons (gluons) goes back to the Low theorem [6, 7], which states that the leading, $\mathcal{O}(1 / w)$ ( $w$ is the photon energy), and next-to-leading, $\mathcal{O}\left(w^{0}\right)$, terms in the radiative amplitude in a generic process can be expressed in terms of the radiationless amplitude. As a consequence, in the soft approximation the amplitude can be written as a product of a universal bremsstrahlung factor times the amplitude that describes the hard process. Under certain conditions, the bremsstrahlung factorization theorem can be extended [8] to include hard collinear radiation with small transverse momentum $k_{t}$ (see also [1]-3] and [9, 10]).

An important consequence of factorization is that the log-enhanced part of the perturbative expansion of differential cross-sections exponentiates. This means that a leading order calculation of the single parton branching probability is sufficient to generate the leading contributions to any order in perturbation theory.

In single-scale observables renormalization group and physical considerations like [11 allow one to choose the renormalization scale $\mu^{2}$ such that unnecessarily large logs are avoided. In differential cross-sections there are several physical scales, e.g. the hard scale $Q^{2}$ and $Q^{2}(1-x)$, so large logs are inherent. The choice of the renormalization point as the hardest scale $\mu^{2} \simeq Q^{2}$ will make the coupling small. However, for $x \longrightarrow$ 1 the cross-section is determined by multiple soft and collinear emission with typical momenta that are much smaller than $Q$. Using $\alpha_{s}\left(Q^{2}\right)$ as an expansion parameter will force the coefficients to be large, reflecting the presence of the lower scale $Q^{2}(1-x)$. As a consequence the resummation of the differential cross-section is strictly restricted to the range $Q^{2}(1-x)>\bar{\Lambda}^{2}$ where $\bar{\Lambda}$ is the scale at which the running coupling diverges. In addition, within the perturbative domain, perturbative and non-perturbative corrections are large.

For fragmentation functions and deep inelastic structure functions, which are both governed by collinear emission, the perturbative domain is $Q^{2}(1-x)>\bar{\Lambda}^{2}$. In event-shape distribution, and in Drell-Yan production close to threshold, large angle soft emission enforces a more stringent restriction, e.g. $Q(1-T)>\bar{\Lambda}$ in the case of the thrust $(T)$. In terms of the coupling, the largest possible domain of applicability of Sudakov resummation is therefore $A L<\xi_{\max }$, where $A \equiv \beta_{0} \alpha_{s}\left(Q^{2}\right) / \pi$ and $\xi_{\max }=1$ or $1 / 2$ in the two cases 
mentioned.

It is well known that perturbative coefficients in QCD increase factorially at large orders. The dominant source of divergence are diagrams that are related to the running coupling, the so-called "renormalons". Due to renormalons, the applicability of fixedorder perturbation theory may be more restricted than a priori expected. The problem can be addressed in perturbation theory by the resummation of the running coupling effects, using the analogy with the Abelian theory [11]-[14].

It is important to realize that a perturbative treatment of running coupling effects is not sufficient: infrared renormalons make the resummation procedure ambiguous at power accuracy. For some inclusive observables, such as the total cross-section in $e^{+} e^{-}$ annihilation, power corrections are small $\sim 1 / Q^{4}$. This can be established by an operator product expansion or by the analysis of infrared renormalon ambiguity [15, 16, 14. On the other hand, in differential cross-sections, the leading corrections are determined by the lower scale, i.e. $\sim 1 /\left(Q^{2}(1-x)\right)^{n}$ (or, for event-shape variables such as the thrust, $\left.1 /(Q(1-T))^{n}\right)$. Consequently also the power-correction expansion is expected to break down for $Q^{2}(1-x) \gtrsim \bar{\Lambda}^{2}$ (or for $Q(1-T) \gtrsim \bar{\Lambda}$ in the thrust case). The positive side is that the factorization property holds beyond the perturbative level. It is natural to expect that like the perturbative series also the power-correction series exponentiates close to the kinematic threshold [17]-23.

The impact of renormalons on differential cross-sections is both interesting in principle and important in practice. The analysis of the thrust distribution $e^{+} e^{-}$annihilation in 23] showed that due to renormalons, sub-leading logs are factorially enhanced with respect to the leading logs. This observation is completely general: this is the way in which sensitivity to lower scales is reflected in the perturbative coefficients. The immediate consequence is that resummation based on a fixed logarithmic accuracy has a limited range of validity, $L A \ll \xi_{\max }$. A quantitative treatment in a wider range (still $L A<\xi_{\max }$ ) requires to sum all the factorially enhanced sub-leading logs and include the associated power corrections in the exponent. This is achieved by Dressed Gluon Exponentiation (DGE) [23].

DGE is designed to deal with both Sudakov logs and renormalons. It is based on exponentiating the entire perturbative series (of log-enhanced terms) that describes the single gluon emission close to the threshold. At a difference from the standard parton cascade approach (see e.g. [4, 24]), the single gluon emission probability is calculated to any order in perturbation theory (in the large- $\beta_{0}$ limit) and to any logarithmic accuracy, thus incorporating the factorial enhancement of sub-leading logs. In addition to the improved perturbative treatment, by identifying the divergence of the exponent one can introduce parametrization of the relevant non-perturbative corrections.

A good example where renormalon analysis is crucial in identifying the leading power corrections is the case of Drell-Yan production [17], [25]-27]. Here, kinematic considerations suggest that as in the thrust distribution, soft gluon emission at large angles will result in a $1 / Q(1-x)$ correction. Renormalon analysis [26, 27] shows that the leading correction is in fact $\sim 1 / Q^{2}(1-x)^{2}$. This difference between the case of event-shape variables and that of Drell-Yan is very intriguing and we shall return to discuss it below.

In both [26] and [23], the starting point for the perturbative calculation was that of the dispersive approach in renormalon calculus [28, 29] (see also [14, 13]): evaluation of 
the single dressed gluon (SDG) cross-section based on the exact matrix element and the exact off-shell gluon phase space. On the other hand, the factorization property and the resulting Sudakov log resummation methodology (see e.g. «, 3, 5, 24]) are based on keeping the gluon on-shell and making specific kinematic approximations that single out the singular parts of the gluon emission matrix element. Although integrals over the running coupling have a central role in both cases, the scale of the coupling in the former is the gluon virtuality, whereas in the latter it is its transverse momentum. Thus at first sight, the two methodologies seem mutually exclusive. In this paper we show how they can be bridged over. We identify the appropriate approximation required to single out the singular part of the matrix element in the case of an off-shell gluon emission. Next, we show that the factorization property applies to off-shell gluons in the same way it applies to on-shell gluons. This allows us to fully exploit the power of factorization: the entire renormalon sum exponentiates.

The calculation of the exponent is, of course, approximate. However, instead of working at a fixed logarithmic accuracy, our approximation is based on the Abelian large- $\beta_{0}$ limit. The usual reason to consider this limit is that it identifies the dependence on the scale of the running coupling and thus also the infrared sensitivity. In the context of exponentiation there is an additional motivation: the large- $\beta_{0}$ limit distinguishes single gluon emission from multiple emission. Since the exponentiation kernel is primarily associated with a single gluon emission, it is natural to evaluate it using this limit. The first step beyond the large- $\beta_{0}$ limit is taken in an unambiguous way by identifying the running coupling with the "gluon bremsstrahlung" effective charge [30, 23]. Using this coupling and the two-loop renormalization group equation is sufficient to guarantee that the DGE resums all the logs (i.e. not only the large- $\beta_{0}$ logs) up to next-to-leading logarithmic accuracy (NLL). Contrary to a fixed logarithmic accuracy resummation, DGE is free of renormalization-scale dependence. Clearly, some residual scale dependence appears beyond the logarithmic approximation upon matching the exponentiated result with fixed-order calculations [23]. However, in the threshold region, when the logarithmically enhanced terms dominate, this effect is negligible.

The logarithmically enhanced cross-section can be calculated from the singular terms in the squared matrix element. Using the light-cone axial gauge we demonstrate that as far as the singular terms are concerned, the emission probability is process-independent. The squared matrix element factorizes into a hard part times a function that depends only on the gluon momentum. The latter is interpreted as a generalized splitting function. The factorization of the cross-section depends on the phase space. Here we consider the case where all energetic particles move in two light-cone directions. Such light-cone kinematics arises in different cases: in $e^{+} e^{-}$these are the two opposite directions of the quark jets, in Drell-Yan production near $x=1$ these are the directions of the two incoming quarks, and in deep inelastic scattering near $x_{\mathrm{Bj}}=1$ one light-cone direction is the direction of the incoming quark, the other that of the outgoing quark. In each of these cases we show how the singularity of the matrix element translates into Sudakov logs through the phasespace integration. It is crucial that the logs emerge only from the limit in which the gluon transverse momentum vanishes (it can be soft or collinear). As a consequence, the relevant terms in the characteristic function can be easily computed. The resulting characteristic 
function is integrated with the running coupling yielding a resummed cross-section that contains all the logarithmic terms associated with a single dressed gluon. This is the kernel for DGE.

The outline of this paper is as follows. In section 2 we use the light-cone gauge to analyse the generic scenario of an off-shell gluon bremsstrahlung in light-cone kinematics. We identify the approximation needed to isolate the singular parts of the squared matrix element and show that in this approximation the radiative squared matrix element can be written in a factorized form in which a universal bremsstrahlung factor multiplies the nonradiative squared matrix element. In section 3 we turn to discuss factorization at the level of the cross-section. Considering first the case of final-state radiation in $e^{+} e^{-}$annihilation we derive a generalized splitting function formula for the case of an off-shell gluon. In section 4 we illustrate the advantage of the axial gauge in this context. Using the case of the quark fragmentation function as an example, sections 5 and 6 describe the two steps in promoting the leading order calculation to a resummed one: in section 5 we show how phase-space integration can be performed within the approximation considered to obtain the relevant part of the SDG characteristic function and then use the dispersive approach to dress the gluon through an integral over the running coupling. In section 6 we show how multiple emission can be taken into account by exponentiation of the SDG cross-section. Sections 7 though 9 summarize the application of the method to other observables: jet mass (or thrust) in $e^{+} e^{-}$, coefficient functions in deep inelastic scattering and DrellYan production. Common features and differences between the different examples are emphasized. Section 10 contains some concluding remarks.

\section{Factorization of the squared matrix element}

Sudakov logs appear because of soft or collinear gluon radiation off nearly on-shell partons. The emitting parton can be a gluon or a quark. In the standard treatment of parton cascades, each possible branching is assigned a probability, which is a function of the longitudinal momentum fraction. Here we develop a similar probabilistic formalism for the case of an off-shell gluon emission off a quark. Gluon splitting will be taken into account in a different way, through an integral over the running coupling.

In a generic QCD process, consider gluon emission off an outgoing quark, assuming that the quark is on-shell after the emission $p^{2}=m_{q}^{2} \simeq 0$, while the gluon possesses a time-like virtuality $k^{2}=m^{2}$. The amplitude for the emission off this particular quark is

$$
\mathcal{M}=g_{s} t^{a} \epsilon_{\mu}^{\lambda^{*}} \frac{1}{(k+p)^{2}} \bar{u}^{(s)}(p) \gamma^{\mu}(\not p+\not k) \mathcal{M}_{r}
$$

where $t^{a}$ is a colour matrix, $\epsilon_{\mu}^{\lambda^{*}}$ is the gluon polarization vector and $\mathcal{M}_{r}$ represents the rest of the process. The enhancement of soft or collinear emission is associated with the singularity of the quark propagator $1 /(k+p)^{2}$, which is only partially compensated by the terms in the numerator. Factorization of the cross-section is based on the fact that the singularity emerges just from this diagram. The squared matrix element corresponding 


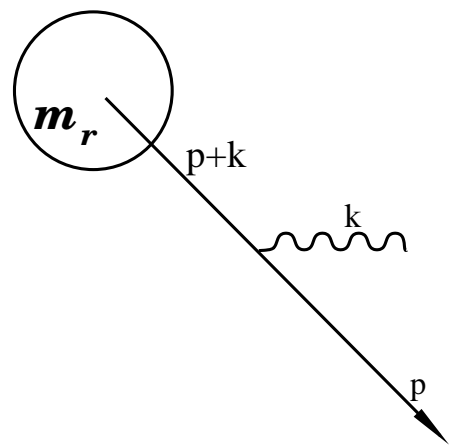

Figure 1: Gluon bremsstrahlung off an outgoing quark

to (1) summed over the gluon and quark spins is

$$
\sum_{\text {spins }} \mathcal{M} \mathcal{M}^{\dagger}=\frac{C_{F} g_{s}^{2}}{(k+p)^{4}}\left(\sum_{\lambda} \epsilon_{\nu}^{\lambda^{*}} \epsilon_{\mu}^{\lambda}\right) \operatorname{Tr}\left\{(\not p+\not k) \gamma^{\nu} \not p \gamma^{\mu}(\not p+\not k) \mathcal{M}_{r} \mathcal{M}_{r}^{\dagger}\right\}
$$

where $\sum_{s} u^{(s)}(p) \bar{u}^{(s)}(p)$ was replaced by $\not p$. Since the gluon attaches also to other particles with an amplitude $\overline{\mathcal{M}}$, the full calculation of the cross-section should be based on $(\mathcal{M}+\overline{\mathcal{M}})(\mathcal{M}+\overline{\mathcal{M}})^{\dagger}$ rather than on (2) . However, the explicit terms in (2) capture the entire singularity if interference terms of the form $\mathcal{M} \overline{\mathcal{M}}^{\dagger}$ are non-singular. This depends on the gauge choice $\sum_{\lambda} \epsilon_{\nu}^{\lambda^{*}} \epsilon_{\mu}^{\lambda} \equiv d_{\mu \nu}$.

Defining two light-like vectors by $p=\left(p_{+}, 0,0\right)$ and $\bar{p}=\left(0, \bar{p}_{-}, 0\right)$, where $p^{2}=\bar{p}^{2}=0$, we choose the light-cone axial gauge where $A_{+}=0$, which is a physical gauge: $g^{\mu \nu} d_{\mu \nu}=-2$. The gluon propagator is given by

$$
d_{\mu \nu}=-g_{\mu \nu}+\frac{k_{\mu} \bar{p}_{\nu}+\bar{p}_{\mu} k_{\nu}}{k \bar{p}}
$$

Let us consider now light-cone kinematics. Particles moving in the "-" direction couple to the gauge field through $J_{-} A_{+}$, where $J_{-}$is an effective current. Since in this gauge $A_{+}$vanishes, these particles do not interact with the gauge field. Therefore, if we assume that the other coloured particles move close enough to the "-" direction, it is sufficient to consider the amplitude in (11). Put differently, in this gauge (2) captures the entire singularity and the interference terms $\mathcal{M} \overline{\mathcal{M}}^{\dagger}$ are non-singular. This point will be demonstrated explicitly below.

Note that the assumption we made here is rather strong: the invariant mass of the particles moving in the "-" direction must be negligible. This assumption is relevant in many applications including event-shape variables close to the two-jet limit, and fragmentation functions, deep inelastic scattering structure functions and Drell-Yan production for $x \longrightarrow 1$. On the other hand, this assumption is inadequate for a generic jet observable in hadron-hadron collisions where both initial- and final-state radiation are important, nor for the case of three jets in $e^{+} e^{-}$annihilation. 
Let us now calculate the squared matrix element (2) in the light-cone gauge. The traces corresponding to the two terms in the propagator (3) are

$$
\begin{aligned}
& r_{1} \equiv 2 \operatorname{Tr}\left\{(\not p+\not k) \not p(\not p+\not h) \mathcal{M}_{r} \mathcal{M}_{r}^{\dagger}\right\} \\
& =-2 m^{2} \operatorname{Tr}\left\{\not p \mathcal{M}_{r} \mathcal{M}_{r}^{\dagger}\right\}+4 p k \operatorname{Tr}\left\{\not k \mathcal{M}_{r} \mathcal{M}_{r}^{\dagger}\right\} \\
& r_{2} \equiv \frac{1}{k \bar{p}}\left[\operatorname{Tr}\left\{(\not p+\not k) \not p \not p \not k(\not p+\not k) \mathcal{M}_{r} \mathcal{M}_{r}^{\dagger}\right\}+\operatorname{Tr}\left\{(\not p+\not k) \not k \not p \not p(\not p+\not k) \mathcal{M}_{r} \mathcal{M}_{r}^{\dagger}\right\}\right] \\
& =\frac{(k+p)^{2}}{k \bar{p}}\left[(4 p \bar{p}+2 k \bar{p}) \operatorname{Tr}\left\{\not p \mathcal{M}_{r} \mathcal{M}_{r}^{\dagger}\right\}+2 p \bar{p} \operatorname{Tr}\left\{\not k \mathcal{M}_{r} \mathcal{M}_{r}^{\dagger}\right\}-2 p k \operatorname{Tr}\left\{\not p \mathcal{M}_{r} \mathcal{M}_{r}^{\dagger}\right\}\right] .
\end{aligned}
$$

Collecting the terms $r \equiv r_{1}+r_{2}$ one obtains

$$
\begin{aligned}
r & =\operatorname{Tr}\left\{\not p \mathcal{M}_{r} \mathcal{M}_{r}^{\dagger}\right\}\left[-2 m^{2}+\frac{(k+p)^{2}}{k \bar{p}}(4 p \bar{p}+2 k \bar{p})\right] \\
& +\operatorname{Tr}\left\{\not k \mathcal{M}_{r} \mathcal{M}_{r}^{\dagger}\right\}\left[4 p k+2 p \bar{p} \frac{(k+p)^{2}}{k \bar{p}}\right]+\operatorname{Tr}\left\{\not p \mathcal{M}_{r} \mathcal{M}_{r}^{\dagger}\right\}\left[\frac{-2 p k(k+p)^{2}}{k \bar{p}}\right] .
\end{aligned}
$$

Introducing the Sudakov decomposition of $k=\left(k_{+}, k_{-}, k_{t}\right)=\beta p+\alpha \bar{p}+k_{t}$, and defining the following dimensionless parameters

$$
\begin{aligned}
\beta & =k_{+} / p_{+}=2 k \bar{p} / 2 p \bar{p} \\
\alpha & =k_{-} / \bar{p}_{-}=2 k p / 2 p \bar{p} \\
\gamma & \equiv k_{t}^{2} / 2 p \bar{p} \\
\lambda & =m^{2} / 2 p \bar{p}=\left(2 k_{+} k_{-}-k_{t}^{2}\right) / 2 p \bar{p}=\alpha \beta-\gamma
\end{aligned}
$$

the trace becomes

$$
\begin{aligned}
r & =2 p \bar{p} \operatorname{Tr}\left\{\not p \mathcal{M}_{r} \mathcal{M}_{r}^{\dagger}\right\}\left[-2 \lambda+2 \frac{\alpha+\lambda}{\beta}(2+\beta)\right] \\
& +2 p \bar{p} \operatorname{Tr}\left\{\not k \mathcal{M}_{r} \mathcal{M}_{r}^{\dagger}\right\}\left[2 \alpha+2 \frac{\alpha+\lambda}{\beta}\right]+2 p \bar{p} \operatorname{Tr}\left\{\not p \mathcal{M}_{r} \mathcal{M}_{r}^{\dagger}\right\}\left[-2 \alpha \frac{\alpha+\lambda}{\beta}\right] .
\end{aligned}
$$

Sudakov logs in the differential cross-section are associated with the region where the denominator of the quark propagator $(p+k)^{2}=2 p k+k^{2}=2 p \bar{p}(\alpha+\lambda)$ is small. Therefore, from now on we assume $\alpha+\lambda \ll 1$. Since both $\alpha$ and $\lambda$ are positive, both must be small. The gluon on-shell condition $\alpha \beta=\gamma+\lambda$ then implies that the transverse momentum fraction $\gamma$ is small, at least with respect to $\beta$. We are therefore interested in the contribution from the region where $\alpha, \lambda$ and $\gamma$ are all small, but no specific hierarchy between them is imposed. This approximation is adequate to treat both the soft and the collinear regions: $\beta$ is small in the soft limit, but it is of order 1 or larger in the collinear (non-soft) limit.

In this approximation one can simplify (17) by

- replacing $\operatorname{Tr}\left\{\not k \mathcal{M}_{r} \mathcal{M}_{r}^{\dagger}\right\}$ by $\beta \operatorname{Tr}\left\{\not p \mathcal{M}_{r} \mathcal{M}_{r}^{\dagger}\right\}$. The other components of $k$ have additional suppression by one of the small parameters. 
- ignoring the terms proportional to $\operatorname{Tr}\left\{\not p \mathcal{M}_{r} \mathcal{M}_{r}^{\dagger}\right\}$. Taking $\bar{p}_{-}$of the order of $p_{+}$, these are explicitly suppressed by $\alpha$ or $\lambda$, with respect to the relevant terms.

Then the following expression for the trace $r$ is obtained,

$$
r=2\left[-\lambda(1+\beta)+(\alpha+\lambda) \frac{\beta^{2}+2 \beta+2}{\beta}\right] 2 p \bar{p} \operatorname{Tr}\left\{\not p \mathcal{M}_{r} \mathcal{M}_{r}^{\dagger}\right\},
$$

and, finally, the squared matrix element (2) is

$$
\sum_{\text {spins }} \mathcal{M M}^{\dagger}=2 C_{F} g_{s}^{2}\left[\frac{-\lambda}{(\alpha+\lambda)^{2}}(1+\beta)+\frac{1}{\alpha+\lambda} \frac{\beta^{2}+2 \beta+2}{\beta}\right] \frac{\operatorname{Tr}\left\{\not p \mathcal{M}_{r} \mathcal{M}_{r}^{\dagger}\right\}}{2 p \bar{p}} .
$$

This demonstrates that as far as the $\alpha+\lambda \longrightarrow 0$ singular terms are concerned, the squared matrix element is factorized into a process-independent factor, corresponding to the emission of an off-shell gluon, times $\operatorname{Tr}\left\{\not p \mathcal{M}_{r} \mathcal{M}_{r}^{\dagger}\right\}$, which is the expression for the squared matrix element in case of no gluon emission. The latter is, of course, processdependent.

In the case of gluon radiation off an incoming quark, similar considerations yield

$$
\frac{1}{2} \sum_{\text {spins }} \mathcal{M M}^{\dagger}=2 C_{F} g_{s}^{2}\left[\frac{-\lambda}{(\alpha-\lambda)^{2}}(1-\beta)+\frac{1}{\alpha-\lambda} \frac{\beta^{2}-2 \beta+2}{\beta}\right] \frac{\frac{1}{2} \operatorname{Tr}\left\{\not p \mathcal{M}_{r} \mathcal{M}_{r}^{\dagger}\right\}}{2 p \bar{p}}
$$

where the factor $1 / 2$ accounts for averaging over the quark spin. This expression can be obtained directly from (9) by taking $\alpha \longrightarrow-\alpha$ and $\beta \longrightarrow-\beta$, corresponding to inverting the momenta $p$ and $\bar{p}$. Note that in (10), as in (9), the Sudakov parameters $\alpha, \beta, \gamma$ and $\lambda$ are all positive.

\section{Gluon emission probability}

To show factorization of the physical cross-section, one has to consider the phase space. Suppose that the external particle momenta are denoted by $p^{i}$ and $p^{f}$ for incoming and outgoing particles, respectively. The cross-section for $p_{A}^{i}+p_{B}^{i} \longrightarrow \sum_{n} p_{n}^{f}+k$ is

$$
\begin{aligned}
\sigma= & \frac{1}{4 E_{A} E_{B}\left|v_{A}-v_{B}\right|} \prod_{n} \int \frac{d^{3} \vec{p}_{n}^{f}}{(2 \pi)^{3}} \frac{1}{2 E_{n}^{f}} \\
& \int \frac{d^{4} k}{(2 \pi)^{3}} \delta\left(k^{2}-m^{2}\right)(2 \pi)^{4} \delta^{(4)}\left(p_{A}^{i}+p_{B}^{i}-\sum_{n} p_{n}^{f}-k\right) \sum_{\text {spins }} \mathcal{M} \mathcal{M}^{\dagger}
\end{aligned}
$$

Because of momentum conservation, the gluon is correlated with the other final state particles. Nevertheless, kinematic factorization can be established for a simple enough phase space in the appropriate Mellin or Laplace space. The details of factorization and the resulting exponentiation formula depend on the observable (see e.g. [4, 5, 24]).

As an example we shall consider here (and in sections 4 through 7) the case of $e^{+} e^{-}$ annihilation into hadrons. It is convenient to identify the vectors that define the " + " and 
"-" directions $p$ and $\bar{p}$ as the final momenta of the two primary quarks. Working in the approximation of independent emission, we can calculate the gluon emission probability as if there are only three outgoing particles $p, \bar{p}$ and $k$. Multiple emission results, in this approximation, in straightforward exponentiation, as described in section 6 . The centreof-mass energy squared is $q^{2}$, where $q \equiv p_{A}^{i}+p_{B}^{i}$. Note that it is not given by $2 p \bar{p}$ : the contribution of gluon momentum cannot be neglected in the collinear limit.

Assuming that factorization occurs, we proceed by performing the integration over $p$ and $\bar{p}$ in the trivial case where $\operatorname{Tr}\left\{\not p \mathcal{M}_{r} \mathcal{M}_{r}^{\dagger}\right\}$ is proportional to $2 p \bar{p}$. The three-particle phase-space integral is then

$$
\begin{array}{r}
\int \frac{d^{4} p}{(2 \pi)^{3}} \delta\left(p^{2}\right) \frac{d^{4} \bar{p}}{(2 \pi)^{3}} \delta\left(\bar{p}^{2}\right) \frac{d^{4} k}{(2 \pi)^{3}} \delta\left(k^{2}-m^{2}\right)(2 \pi)^{4} \delta^{4}(q-p-\bar{p}-k)= \\
\frac{q^{2}}{128 \pi^{3}} \int d \alpha d \beta \frac{1-\lambda}{(1+\alpha+\beta+\lambda)^{3}} \simeq \frac{q^{2}}{128 \pi^{3}} \int d \alpha d \beta \frac{1}{(1+\beta)^{3}}
\end{array}
$$

while the two particle phase-space integral is

$$
\int \frac{d^{4} p}{(2 \pi)^{3}} \delta\left(p^{2}\right) \frac{d^{4} \bar{p}}{(2 \pi)^{3}} \delta\left(\bar{p}^{2}\right)(2 \pi)^{4} \delta^{4}(q-p-\bar{p})=\frac{1}{8 \pi}
$$

Starting with the squared matrix element (9) integrated over the three-particle phase space, we now identify the gluon emission probability as the factor multiplying the crosssection for no gluon emission (which is $\operatorname{Tr}\left\{\not p \mathcal{M}_{r} \mathcal{M}_{r}^{\dagger}\right\}$ integrated over the two-particle phase space). Changing variables from $\lambda$ to

$$
\epsilon \equiv \frac{m^{2}}{Q^{2}}=\frac{\lambda(2 p \bar{p})}{Q^{2}}=\frac{\lambda}{1+\alpha+\beta+\lambda} \simeq \frac{\lambda}{1+\beta}
$$

guarantees that the integration is performed for fixed $m^{2}$ and $Q^{2}$. The gluon emission probability is given by

$$
d P=\frac{C_{F} \alpha_{s}}{2 \pi}\left[\frac{-\lambda}{(\alpha+\lambda)^{2}}(1+\beta)+\frac{1}{\alpha+\lambda} \frac{\beta^{2}+2 \beta+2}{\beta}\right] \frac{d \alpha d \beta d \lambda \delta(\lambda-\epsilon(1+\beta))}{(1+\beta)^{3}} .
$$

Equation (15) can be interpretated as a generalized splitting function for the off-shell gluon case. The standard splitting function can be obtained in the limit $\lambda=0$ : substituting $z=1 /(1+\beta)$ as the longitudinal momentum fraction of the quark, one recovers the standard formula

$$
d P=\frac{C_{F} \alpha_{s}}{2 \pi} \frac{1+z^{2}}{1-z} d z d \ln \alpha .
$$

A further assumption that $\beta \ll 1$ leads to the double logarithmic approximation.

Like the standard on-shell splitting function, eq. (15) should be understood as an evolution kernel, so that multiple branching is described by iteration. This is achieved by DGE [23]. 


\section{Gauge choice and interference}

Before proceeding with the application of eq. (15), it is worth while to have a second look at the matrix element in order to appreciate the significance of the gauge choice. While the following discussion is quite general, to be concrete we will keep on using the example of $e^{+} e^{-} \stackrel{\gamma}{\longrightarrow} q \bar{q} g$. Considering the hadronic tensor $H_{\nu \rho}$, the rest of the amplitude in (11) is $\mathcal{M}_{r}=\gamma^{\nu} u(\bar{p})$, so the process-dependent trace in (9) becomes

$$
\sum_{\text {spins }} \operatorname{Tr}\left\{\not p \mathcal{M}_{r} \mathcal{M}_{r}^{\dagger}\right\}=\operatorname{Tr}\left\{\not p \gamma^{\nu} \not \not \gamma^{\rho}\right\}
$$

If the angle with respect the electron beam is integrated over, as in the total fragmentation function case, it is sufficient to project $H_{\nu \rho}$ on $-g^{\nu \rho}$. Then one can replace $\operatorname{Tr}\left\{\not p \gamma^{\nu} \not p \gamma^{\rho}\right\}$ by $8 p \bar{p}$. The light-cone gauge result for the squared matrix element, based on gluon attachment to the $p$ quark only, is therefore

$$
\left.\sum_{\text {spins }} \mathcal{M} \mathcal{M}^{\dagger}\right|_{\text {light-cone }}=a_{0}\left[\frac{-\lambda}{(\alpha+\lambda)^{2}}(1+\beta)+\frac{1}{\alpha+\lambda} \frac{\beta^{2}+2 \beta+2}{\beta}\right],
$$

where $a_{0} \equiv 8 C_{F} g_{s}^{2}$, but since the gluon attaches also to the antiquark $\bar{p}$ with an amplitude

$$
\overline{\mathcal{M}}=g_{s} t^{a} \epsilon_{\mu}^{\lambda^{*}} \frac{1}{(k+\bar{p})^{2}} \bar{u}^{(s)}(p) \gamma^{\nu}(\not p+\not k) \gamma^{\mu} u^{(s)}(\bar{p})
$$

the full squared matrix element is

$$
\sum_{\text {spins }}\left[\mathcal{M} \mathcal{M}^{\dagger}+\mathcal{M} \overline{\mathcal{M}}^{\dagger}+\overline{\mathcal{M}} \mathcal{M}^{\dagger}+\overline{\mathcal{M}} \overline{\mathcal{M}}^{\dagger}\right]=a_{0}\left[\frac{\alpha \beta-\lambda}{(\alpha+\lambda)^{2}}+2 \frac{1+\alpha+\beta+2 \lambda}{(\alpha+\lambda)(\beta+\lambda)}+\frac{\beta \alpha-\lambda}{(\beta+\lambda)^{2}}\right]
$$

This result is of course gauge-invariant. Let us now examine the origin of the various terms, in particular the singular terms for $\alpha+\lambda \longrightarrow 0$, in different gauges. Let us compare the Feynman gauge and the light-cone gauge. In the Feynman gauge, $\mathcal{M M}^{\dagger}$ is the first term in (19) and the interference is responsible for middle term there. Thus in this gauge only part of the singularity is captured by a calculation $\left(\mathcal{M M}^{\dagger}\right)$ that ignores the possibility of gluon attachment to the rest of the process. On the other hand in the lightcone gauge the entire $\alpha+\lambda \longrightarrow 0$ singularity is captured by (17) while the interference term is

$$
\sum_{\text {spins }}\left[\mathcal{M} \overline{\mathcal{M}}^{\dagger}+\overline{\mathcal{M}} \mathcal{M}^{\dagger}\right]=a_{0}\left[2 \frac{1+\alpha+\beta+2 \lambda}{(\alpha+\lambda)(\beta+\lambda)}-2 \frac{1+\beta}{(\alpha+\lambda) \beta}\right]
$$

which is not singular. This shows that light-cone gauge is indeed useful in identifying the $\alpha+\lambda \longrightarrow 0$ singular terms.

It is also interesting to note the different source of the singularity of the squared matrix element in the double logarithmic approximation. In the Feynman gauge the singularity originates in the interference term: $1 / \alpha$ appears because of the singularity of the propagator of the quark with momentum $p+k$, and $1 / \beta$ from the one with momentum $\bar{p}+k$. In the axial gauge the singularity originates only from the diagram where the gluon interacts with $p$, so $1 / \alpha$ has the same source - the singularity of the propagator. On the other hand, $1 / \beta$ is just built into the gauge (3). 


\section{Dressing the gluon}

Let us demonstrate the application of the off-shell gluon splitting function for DGE. We begin with the simplest example: the case of the massless quark fragmentation in $e^{+} e^{-}$ annihilation. The fragmentation functions were analysed in the dispersive approach by Dasgupta and Webber [32], who concentrated on the determination of the $x$ dependence of the power corrections. Our results for SDG characteristic function in the limit $x \longrightarrow 1$ are consistent with their calculation. The main difference between our approach and that of 29, 32] is that in the latter the power corrections are additive to the coefficient function, while here (similar ideas were raised before, see [18]- 22], [26, 27], 441] and [23]), as we shall see in the next section, the SDG calculation is considered as the kernel of exponentiation, so the power corrections will appear as a function that is convoluted with the resummed coefficient function.

The fragmentation functions are defined by

$$
\frac{1}{\sigma} \frac{d \sigma}{d x d \cos \theta}=\frac{3}{8}\left(1+\cos ^{2} \theta\right) F_{T}\left(x, Q^{2}\right)+\frac{3}{4} \sin ^{2} \theta F_{L}\left(x, Q^{2}\right)+\frac{3}{4} \cos \theta F_{A}\left(x, Q^{2}\right),
$$

where $x \equiv 2 q p_{h} / Q^{2}$. Here $q$ is the electroweak gauge boson momentum $\left(q^{2} \equiv Q^{2}>0\right)$, $p_{h}$ is the hadron momentum and $\theta$ is the angle of the hadron with respect to the $e^{-}$ beam; $F_{T}\left(x, Q^{2}\right), F_{L}\left(x, Q^{2}\right)$ and $F_{A}\left(x, Q^{2}\right)$ correspond to the transverse, longitudinal and asymmetric fragmentation functions, respectively. $F_{A}\left(x, Q^{2}\right)$ vanishes for a pure electromagnetic interaction. The standard factorization technique allows one to compute the coefficient function $C_{P}^{i}\left(z, Q^{2}\right)$ by identifying the outgoing hadron as an outgoing parton $i$. The physical fragmentation function is then written as a convolution with the non-perturbative function $D\left(x, Q^{2}\right)$,

$$
F_{P}\left(x, Q^{2}\right)=\sum_{i} \int_{x}^{1} \frac{d z}{z} C_{P}^{i}\left(z, Q^{2}\right) D_{i}\left(x / z, Q^{2}\right),
$$

where $i$ can be a quark, an antiquark or a gluon. In moment space,

$$
\int_{0}^{1} F_{P}\left(x, Q^{2}\right) x^{N-1} d x \equiv F_{P}\left(N, Q^{2}\right)
$$

the convolution translates into a product

$$
F_{P}\left(N, Q^{2}\right)=\sum_{i} C_{P}^{i}\left(N, Q^{2}\right) D_{i}\left(N, Q^{2}\right)
$$

We will now show that the log-enhanced terms in the quark fragmentation coefficient function $C^{q}\left(x, Q^{2}\right)$, which are associated with a single gluon emission, can be directly calculated from (15). In the next section this result will be used to calculate the multiple gluon (resummed) coefficient function through DGE.

The gluon virtuality $\epsilon$ has a crucial role in the calculation of the single gluon emission cross-section to all orders. For the calculation to be correct to any logarithmic accuracy

\footnotetext{
${ }^{\dagger}$ A perturbative analysis at NLL was recently completed 31] for the case of heavy quark fragmentation. Here we discuss only the massless case.
} 
(even in the simplest case, i.e. the large- $N_{f}$ limit), an integral over the running coupling must be performed with the correct argument - the gluon virtuality. This is the physical scale of the interaction. Using the dispersive approach in renormalon calculus, the integral over the running coupling is equivalent to the sum of the perturbative series in the large$\beta_{0}$ limit. Such a renormalon calculation can be performed within the approximation considered in the derivation of (15), where only the singular terms in the matrix element were kept. This approximation amounts to keeping all the logs in the differential crosssection, while neglecting terms that are explicitly suppressed by $1-x$.

The total quark fragmentation function $F\left(x, Q^{2}\right)=F_{T}\left(x, Q^{2}\right)+F_{L}\left(x, Q^{2}\right)$ is certainly sensitive to soft or collinear emission - we saw that the squared matrix element (17) (or (19)) is singular for $\alpha+\lambda \longrightarrow 0$. The quark that emits the gluon (in our gauge) is $p$. We therefore identify $p_{h}$ as $\bar{p}$, getting

$$
x \equiv \frac{2 \bar{p} q}{q^{2}}=\frac{1+\beta}{1+\alpha+\beta+\lambda} .
$$

We see that the singularity in (15) is inversely proportional to $1-x \simeq(\alpha+\lambda) /(1+\beta)$. Using this variable, the differential gluon emission probability is

$$
\frac{d P}{d x}=\frac{C_{F} \alpha_{s}}{2 \pi} \int d \beta\left[-\frac{\epsilon}{(1-x)^{2}} \frac{1}{(1+\beta)^{2}}+\frac{1}{1-x} \frac{\beta^{2}+2 \beta+2}{\beta(1+\beta)^{3}}\right],
$$

where $\epsilon=m^{2} / Q^{2}$. In the integration over $\beta$, the limit that contributes to log-enhanced terms is the limit where the gluon transverse momentum $\gamma$ vanishes. This translates into

$$
\beta=\lambda / \alpha=\epsilon /(1-x-\epsilon)
$$

We therefore integrate over $\beta$ and substitute (26), getting

$$
\frac{d P}{d x}=\frac{C_{F} \alpha_{s}}{2 \pi} \mathcal{F}(x, \epsilon)
$$

with

$$
\left.\mathcal{F}(x, \epsilon)\right|_{\log }=\frac{2}{1-x} \ln \left(\frac{1-x}{\epsilon}\right)-\frac{3}{2} \frac{1}{1-x}+\frac{\epsilon}{(1-x)^{2}}+\frac{1}{2} \frac{\epsilon^{2}}{(1-x)^{3}} .
$$

The physical region is $\epsilon<1-x$, where $\alpha$ and $\beta$ are positive; $\mathcal{F}(x, \epsilon)$ is the off-shell gluon characteristic function [29, 32, 33] for the quark fragmentation function. The subscript ' $\log$ ' in (28) is meant to remind us of the approximation: by taking only the $1-x \longrightarrow 0$ singular terms in the matrix element, we control only log-enhanced terms in the perturbative coefficients. The full characteristic function (see e.g. [32]) contains some additional terms, which generate non-logarithmic terms in the perturbative series.

\footnotetext{
${ }^{\ddagger}$ Here we dropped sub-leading terms (e.g. $\left.\mathcal{O}\left(\epsilon^{2} /(1-x)^{2}\right)\right)$, which go beyond our approximation and contribute only to non-logarithmic terms in the perturbative coefficients.
} 
In the dispersive approach [29] (see also [28, 14, 13]) the single off-shell gluon result (27) is promoted to an infinite perturbative-sum associated with a single dressed gluon by replacing the coupling with a dispersive integral

$$
\frac{C_{F}}{2 \beta_{0}} \int_{0}^{1-x} \frac{d \epsilon}{\epsilon} \dot{\mathcal{F}}(x, \epsilon) \bar{A}_{\text {eff }}\left(\epsilon Q^{2}\right)=\frac{C_{F}}{2 \beta_{0}} \int_{0}^{1-x} \frac{d \epsilon}{\epsilon}[\mathcal{F}(\epsilon)-\mathcal{F}(0)] \bar{\rho}\left(\epsilon Q^{2}\right),
$$

where $\bar{A}\left(k^{2}\right) \equiv \beta_{0} \bar{\alpha}_{s}\left(k^{2}\right) / \pi$. The two integrals in (29) are related by integration by parts: $\dot{\mathcal{F}}(x, \epsilon) \equiv-\epsilon d \mathcal{F}(x, \epsilon) / d \epsilon$, and the function $\bar{\rho}\left(\mu^{2}\right)$ is identified as the time-like discontinuity of the coupling,

$$
\bar{\rho}\left(\mu^{2}\right)=\frac{1}{2 \pi i} \operatorname{Disc}\left\{\bar{A}\left(-\mu^{2}\right)\right\} \equiv \frac{1}{2 \pi i}\left[\bar{A}\left(-\mu^{2}+i \lambda\right)-\bar{A}\left(-\mu^{2}-i \lambda\right)\right] .
$$

The "time-like coupling" $\bar{A}_{\text {eff }}\left(\mu^{2}\right)$ in (29) obeys $\mu^{2} d \bar{A}_{\text {eff }}\left(\mu^{2}\right) / d \mu^{2}=\bar{\rho}\left(\mu^{2}\right)$. For example, in the one-loop case $\bar{A}\left(k^{2}\right)=1 /\left(\ln k^{2} / \bar{\Lambda}^{2}\right)$ and

$$
\bar{A}_{\text {eff }}\left(\mu^{2}\right)=\frac{1}{2}-\frac{1}{\pi} \arctan \left(\frac{1}{\pi} \ln \frac{\mu^{2}}{\bar{\Lambda}^{2}}\right) .
$$

We emphasize that (29) is considered just as a perturbative sum, and not as a nonperturbative definitions.

The bar over the coupling indicates a specific renormalization scheme. In the large- $\beta_{0}$ limit (large- $N_{f}$ limit) $\bar{A}\left(k^{2}\right)$ is related to the $\overline{\mathrm{MS}}$ coupling $A_{\overline{\mathrm{MS}}}\left(k^{2}\right) \equiv \beta_{0} \alpha_{s}^{\overline{\mathrm{MS}}}\left(k^{2}\right) / \pi$ by $\bar{A}\left(k^{2}\right)=A_{\overline{\mathrm{MS}}}\left(k^{2}\right) /\left(1-\frac{5}{3} A_{\overline{\mathrm{MS}}}\left(k^{2}\right)\right)$. In the general case, going beyond this limit requires a skeleton expansion [12], generalizing the Abelian one. However, in the context of our approximation, where only the singularity of the splitting function matters, a skeleton expansion is not required. The appropriate coupling is the "gluon bremsstrahlung" effective charge [30, 23]. Fixing $\bar{A}$ by

$$
\bar{A}\left(k^{2}\right)=\frac{A_{\overline{\mathrm{MS}}}\left(k^{2}\right)}{1-\left[\frac{5}{3}+\left(\frac{1}{3}-\frac{\pi^{2}}{12}\right) C_{A} / \beta_{0}\right] A_{\overline{\mathrm{MS}}}\left(k^{2}\right)}
$$

the splitting function (15) is correct to next-to-leading order, as far as the singular terms are concerned. The origin of the "gluon bremsstrahlung" effective charge can be understood by the formulation of the Sudakov resummation through an evolution equation for Wilson-line operators. In this language this effective charge appears as the cusp anomalous dimension [34, 35].

In the quark fragmentation case (28), we have

$$
\left.\dot{\mathcal{F}}(x, \epsilon)\right|_{\log }=\frac{2}{1-x}-\frac{\epsilon}{(1-x)^{2}}-\frac{\epsilon^{2}}{(1-x)^{3}} .
$$

Note that $\dot{\mathcal{F}}(x, \epsilon=0) \neq 0$ so the integral $(29)$ diverges for vanishing gluon virtuality. This is a direct consequence of the fact that the fragmentation function is not collinear-safe.

\footnotetext{
${ }^{\S}$ Such a definition differs from the Borel-sum by power-corrections which are not associated with the infrared dynamics 13, 28, 14.
} 
Factorization (22) is required. We shall make the following identification: $C^{q}\left(x, Q^{2}\right)=$ $\delta(1-x)+C_{\mathrm{SDG}}^{q}\left(x, Q^{2}\right)+\ldots$ where the ellipses stand for corrections that are sub-leading by powers of either $\beta_{0}$ or $1-x$ and

$$
\begin{gathered}
C_{\mathrm{SDG}}^{q}\left(x, Q^{2}\right)=\frac{C_{F}}{2 \beta_{0}}\left[\int_{1}^{1-x} \frac{d \epsilon}{\epsilon} \mathcal{S}(x) \bar{A}_{\mathrm{eff}}\left(\epsilon Q^{2}\right)+\int_{0}^{1-x} \frac{d \epsilon}{\epsilon}(\dot{\mathcal{F}}(x, \epsilon)-\mathcal{S}(x)) \bar{A}_{\mathrm{eff}}\left(\epsilon Q^{2}\right)\right] \\
=\frac{C_{F}}{2 \beta_{0}}\left[\frac{2}{1-x} \int_{1}^{1-x} \frac{d \epsilon}{\epsilon} \bar{A}_{\mathrm{eff}}\left(\epsilon Q^{2}\right)-\int_{0}^{1-x} \frac{d \epsilon}{\epsilon}\left(\frac{\epsilon}{(1-x)^{2}}+\frac{\epsilon^{2}}{(1-x)^{3}}\right) \bar{A}_{\mathrm{eff}}\left(\epsilon Q^{2}\right)\right] .
\end{gathered}
$$

Here $\mathcal{S}(x)=2 /(1-x)$ is the coefficient of $\ln (1 / \epsilon)$ in $\mathcal{F}(x, \epsilon)$. The subtracted term is absorbed into the non-perturbative function $D_{q}\left(x / z, Q^{2}\right)$. This means that its evolution is controlled by $\mathcal{S}(x)$, i.e. by $C_{F}\left(\bar{\alpha}_{s} / \pi\right) /(1-x)$. With the definition (33) the coefficient function has a well-defined perturbative expansion. Expanding the coupling $\bar{A}_{\text {eff }}\left(\epsilon Q^{2}\right)$ in a fixed-scale coupling one can write the corresponding perturbative coefficients in terms of the log-moments:

$$
\begin{aligned}
h_{n}(x) & \equiv \int_{1}^{1-x} \frac{d \epsilon}{\epsilon}\left(\ln \frac{1}{\epsilon}\right)^{n} \mathcal{S}(x)+\int_{0}^{1-x} \frac{d \epsilon}{\epsilon}\left(\ln \frac{1}{\epsilon}\right)^{n}(\dot{\mathcal{F}}(x, \epsilon)-\mathcal{S}(x)) \\
& =\frac{-1}{(1-x)}\left[\frac{2}{n+1} L^{n+1}+e^{L} \Gamma(n+1, L)+2^{-n-1} e^{2 L} \Gamma(n+1,2 L)\right],
\end{aligned}
$$

where $L=\ln 1 /(1-x)$. Since $e^{L} \Gamma(n+1, L)=\sum_{j=0}^{n} L^{j} n ! / j !$, the expression in the square brackets is just a polynomial of order $n+1$ in $L$. Note, however, that since the sub-leading logs increase factorially, the sum is not well approximated by the leading log term even at rather large $L$ (see [23]).

Contrary to the total fragmentation function, the longitudinal fragmentation function $F_{L}\left(x, Q^{2}\right)$ is insensitive to soft or collinear emission (at the logarithmic level): projecting (16) onto $\epsilon_{L}^{\nu} \epsilon_{L}^{\rho}$, where

$$
\epsilon_{L}^{\nu} \equiv \frac{1}{|\overline{\mathbf{p}}|}\left(\bar{p}^{\nu}-\frac{q \bar{p}}{q^{2}} q^{\nu}\right)
$$

is the polarization along the direction of $\overline{\mathbf{p}}$, the contribution vanishes. It follows that in the electromagnetic case the log-enhanced terms in the total fragmentation function all originate in the transverse fragmentation function (see [32, 33]). Exactly the same log-enhanced terms appear in the parity-violating asymmetric fragmentation function.

\section{Dressed gluon exponentiation}

We saw that factorization of the squared matrix element, which is the basis for exponentiation, holds also for an off-shell gluon. Exponentiation can be derived, as in [5, 24], from an evolution equation, or, as in [23], directly from the probabilistic interpretation of the SDG cross-section based on the approximation of independent emission.

The factorization of the squared matrix element suggests that the entire log-enhanced SDG sum exponentiates. In reality straightforward exponentiation holds only up to a 
certain logarithmic accuracy, because gluons are correlated through the phase-space integration. Momentum conservation translate into a factorized form in Laplace space (see e.g. [24, 23]). To some logarithmic accuracy one can then perform the integration over the gluon momenta as if other gluons had not been emitted. At the same time exponentiation of the entire SDG sum can be regarded as the leading contribution to the exponent in the large- $\beta_{0}$ limit. In this sense the expansion in powers of $1 / \beta_{0}$ in the exponent replaces the standard expansion in powers of the log.

Being aware of this we now proceed to write down the explicit exponentiation formula for the fragmentation function case. We work in the approximation where the invariant mass of the jet, $Q^{2}(1-x)$, is additive with respect to multiple gluon emission. With this assumption we ignore correlations between the gluons, which would modify the exponentiation beyond NLL accuracy. The resummed expression for $C^{q}\left(x, Q^{2}\right)$ is then given by the inverse Mellin transform of $\left.C_{q}\left(N, Q^{2}\right)\right|_{\text {res }}$, where

$$
\left.C_{q}\left(N, Q^{2}\right)\right|_{\mathrm{res}}=\exp \left\{\int_{0}^{1} d x\left(x^{N-1}-1\right) C_{\mathrm{SDG}}^{q}\left(x, Q^{2}\right)\right\} .
$$

Here we drop an $x$-independent factor associated with the virtual corrections to the hard cross-section (see e.g. [31]).

A convenient way to deal with perturbation theory to all orders is Borel summation. Starting with the scheme-invariant Borel representation of the coupling [36]-38],

$$
\bar{A}\left(k^{2}\right)=\int_{0}^{\infty} d u \exp \left(-u \ln k^{2} / \Lambda^{2}\right) \bar{A}_{B}(u)
$$

and taking the time-like discontinuity [36] we get

$$
\bar{A}_{\mathrm{eff}}\left(Q^{2}\right)=\int_{0}^{\infty} d u \exp \left(-u \ln Q^{2} / \Lambda^{2}\right) \frac{\sin \pi u}{\pi u} \bar{A}_{B}(u)
$$

Using this Borel representation of $\bar{A}_{\text {eff }}\left(\epsilon Q^{2}\right)$, and integrating (33) over $\epsilon$, one obtains a Borel integral representing the SDG perturbative sum:

$$
C_{\mathrm{SDG}}^{q}\left(x, Q^{2}\right)=\frac{C_{F}}{2 \beta_{0}} \int_{0}^{\infty} d u B_{\mathrm{SDG}}(u, x) \exp \left(-u \ln Q^{2} / \Lambda^{2}\right) \frac{\sin \pi u}{\pi u} \bar{A}_{B}(u)
$$

with the following Borel function

$$
B_{\mathrm{SDG}}(u, x)=\frac{-1}{1-x}\left[\frac{2\left((1-x)^{-u}-1\right)}{u}+\frac{(1-x)^{-u}}{1-u}+\frac{(1-x)^{-u}}{2-u}\right] .
$$

Next, the exponent in Mellin space $\left.\ln C_{q}\left(N, Q^{2}\right)\right|_{\text {res }}$ is obtained from (35) integrating over $x$

$$
\left.\ln C_{q}\left(N, Q^{2}\right)\right|_{\text {res }}=\frac{C_{F}}{2 \beta_{0}} \int_{0}^{\infty} d u B_{N}(u) \exp \left(-u \ln Q^{2} / \Lambda^{2}\right) \frac{\sin \pi u}{\pi u} \bar{A}_{B}(u)
$$

with

$$
B_{N}(u)=\frac{-2}{u}\left[e^{u \ln N} \Gamma(-u)+\frac{1}{u}+\gamma_{E}+\ln N\right]-\left(\frac{1}{1-u}+\frac{1}{2-u}\right)\left[e^{u \ln N} \Gamma(-u)+\frac{1}{u}\right]
$$

\footnotetext{
IUpon integration $\Gamma(-u)$ appears with a factor $\Gamma(N) / \Gamma(N-u)$. In the approximation considered $(N \gg 1)$ this factor can be replaced by $e^{u \ln N}$.
} 
The Borel function (41) contains valuable information on both the perturbative and the non-perturbative levels.

Sudakov logs: To get an explicit perturbative expansion of the exponent in Mellin space one can perform the Borel integral term by term, similarly to what was done in [23] for the case of the thrust distribution. For simplicity we work with a one-loop running coupling $\bar{A}\left(Q^{2}\right)$. It is straightforward to rewrite the sum as

$$
\left.\ln C_{q}\left(N, Q^{2}\right)\right|_{\mathrm{res}}=\frac{C_{F}}{2 \beta_{0}} \sum_{k=1}^{\infty} \bar{A}\left(Q^{2}\right)^{k-2} f_{k}\left(\bar{A}\left(Q^{2}\right) \ln N\right),
$$

where the functions $f_{k}(\xi)$ sum all powers of $\xi \equiv \bar{A}\left(Q^{2}\right) \ln N$. They are given by

$$
f_{k}(\xi)=\sum_{l=0}^{(k-1) / 2} \frac{\left(-\pi^{2}\right)^{l}}{(2 l+1) !} g_{k}^{(l)}(\xi) M_{\mathrm{col}}(k),
$$

where $g_{k}^{(l)}(\xi)$ is the following hypergeometric function

$$
g_{k}^{(l)}(\xi)=\xi^{n_{0}} \frac{\Gamma\left(k-2+n_{0}\right)}{\Gamma\left(1+n_{0}\right)}{ }_{2} F_{1}\left(\left[1, k-2+n_{0}\right],\left[1+n_{0}\right], \xi\right)
$$

with $n_{0}=\max (0,2 l+3-k)$ and

$$
M_{\mathrm{col}}(k)=2 c_{k-2}+\sum_{m=0}^{k-2}\left(1+2^{-m-1}\right) c_{k-3-m},
$$

where the numbers $c_{k}$ are defined by $\Gamma(-z)=-\sum_{k=-1}^{\infty} c_{k} z^{k}$. This means that $c_{-1}=1$, $c_{0}=\gamma_{E}, c_{1}=\pi^{2} / 12+\gamma_{E}^{2} / 2$, etc. Higher $c_{k}$ contain higher $\zeta_{i}$ numbers $(i \leq k+1)$, yet numerically $c_{k}(k \geq 1)$ are all close to 1 . For $k=1,2$ the functions are

$$
\begin{aligned}
& f_{1}(\xi)=2(\xi+(1-\xi) \ln (1-\xi)) \\
& f_{2}(\xi)=-\left(\frac{3}{2}+2 \gamma_{E}\right) \ln (1-\xi)
\end{aligned}
$$

while for $k \geq 3$ they are characterized by increasing coefficients as well as increasing order pole singularities at $\xi=1$,

$$
\begin{aligned}
& f_{3}(\xi)=0.804 /(1-\xi)+3.29 \xi \\
& f_{4}(\xi)=0.779 /(1-\xi)^{2} \\
& f_{5}(\xi)=2.32 /(1-\xi)^{3}-9.74 \xi \\
& f_{6}(\xi)=6.12 /(1-\xi)^{4} \\
& f_{7}(\xi)=23.8 /(1-\xi)^{5}+45.78 \xi \\
& f_{8}(\xi)=120.9 /(1-\xi)^{6} \\
& f_{9}(\xi)=721.54 /(1-\xi)^{7}-263.57 \xi \\
& f_{10}(\xi)=5043.48 /(1-\xi)^{8} .
\end{aligned}
$$

We see that sub-leading logs are characterized by an explicit factorial growth as well as by an increasing singularity at the end of the perturbative region:

$$
\xi \equiv \frac{\alpha_{s} \beta_{0}}{\pi} \ln \frac{1}{1-x}=\xi_{\max }
$$


where $\xi_{\max }=1$. A similar structure was identified in the case of the thrust (or jet mass) distribution in [23]. The two cases are compared in the next section. As discussed in [23] the enhancement of sub-leading logs implies that an approximation based on a fixed logarithmic accuracy in the exponent has a small range of validity, namely $\xi \ll \xi_{\max }$. The resummed exponent (40) is valid much closer to the strict limit of applicability of perturbation theory $\left(\xi \lesssim \xi_{\max }\right)$, provided appropriate power corrections are included.

Power corrections: The form of the power corrections can be deduced from the renormalon ambiguity. They are expected to be additive at the level of (40) and therefore they exponentiate together with the perturbative sum, so that

$$
C_{q}\left(N, Q^{2}\right)=\left.C_{q}\left(N, Q^{2}\right)\right|_{\text {res }} C_{q}^{\mathrm{NP}}\left(N, Q^{2}\right)
$$

This implies that the correction can be written as a convolution in $x$ space. The Borel singularity of the fragmentation function exponent in the large- $\beta_{0}$ limit can be read off eq. (41), taking into account the attenuation by the $\sin (\pi u)$ factor in (40). The result turns out to be very simple: a pole at $z=1$ with a residue $-N$ and second pole at $z=2$ with a residue $-N^{2} / 4$. The non-perturbative correction $C_{q}^{\mathrm{NP}}\left(N, Q^{2}\right)$ is therefore expected to be

$$
C_{q}^{\mathrm{NP}}\left(N, Q^{2}\right)=\exp \left\{-\omega_{1} \frac{C_{F}}{2 \beta_{0}} \frac{N \bar{\Lambda}^{2}}{Q^{2}}-\omega_{2} \frac{C_{F}}{2 \beta_{0}} \frac{N^{2} \bar{\Lambda}^{4}}{4 Q^{4}}\right\},
$$

where $\omega_{n}$ are dimensionless constants. Since $C_{q}\left(N, Q^{2}\right)$ multiplies a non-perturbative distribution $D_{q}\left(N, Q^{2}\right)$, eq. (50) leads to a meaningful prediction only if it dominates the behaviour in the threshold region. This possibility should be tested experimentally. As discussed in the next section, the situation is different for collinear- and infrared-safe quantities where no non-perturbative distribution is needed a priori.

\section{Jet mass in $e^{+} e^{-}$annihilation}

One of the classical examples where soft and collinear gluon radiation is important is the case of event-shape variables in $e^{+} e^{-}$annihilation. As opposed to the fragmentation function case, these observables are infrared- and collinear-safe, so the perturbative calculation does not require factorization. The enhanced sensitivity of these observables to large angle soft emission is reflected both perturbatively - as large logs [24], and non-perturbatively - as power corrections [13, 23] (see also [29], [39]- [43] and [18]-[22]).

Here we consider event-shape variables that are related to the jet mass distribution, such as the thrust and the heavy jet mass. The thrust distribution was analysed in detail, using DGE, in [23]. Analysis of the heavy jet mass by the same method will appear soon [44]. The starting point in [23] was the full SDG characteristic function [43]. We shall now see how the relevant part of the characteristic function, the one that generates the logs, can be computed directly from the generalized off-shell splitting function. This will be followed by a brief description of the results of [23] for comparison with other cases studied here.

Consider a single off-shell gluon emission, which is either soft or collinear (two-jet kinematics). Assuming that the gluon is in the hemisphere of the quark $p$, it is $\bar{p}$ that 
sets the thrust axis. The $\bar{p}$ jet mass is zero and the $p$ jet mass, which is also $1-$ thrust, is simply

$$
\rho=\frac{m_{H}^{2}}{Q^{2}}=\frac{(p+k)^{2}}{Q^{2}}=\frac{2 p k+k^{2}}{Q^{2}}=\frac{(\alpha+\lambda) 2 p \bar{p}}{Q^{2}}=\frac{\alpha+\lambda}{1+\alpha+\beta+\lambda}=1-x .
$$

Thus calculation of the SDG characteristic function (neglecting non-inclusive effects 29 , 13, 43, 23, associated with gluon decay into opposite hemispheres) is just identical to that of the total fragmentation function. The result is (32), with the replacement of $1-x$ by $\rho$ :

$$
\left.\dot{\mathcal{F}}(\rho, \epsilon)\right|_{\log }=\frac{2}{\rho}-\frac{\epsilon}{\rho^{2}}-\frac{\epsilon^{2}}{\rho^{3}} .
$$

This result is confirmed by the explicit calculation of the full characteristic function [43] followed by the identification of terms that contribute to logs [23].

A major difference between jet mass distribution and the fragmentation function case is in the large-angle phase-space limit: we assumed that the gluon is in the hemisphere of the quark $p$. This is equivalent to $\beta>\alpha$. Along the zero transverse momentum boundary of phase space $\alpha \beta=\lambda$, this condition translates into $\beta>\sqrt{\lambda}$. It follows that the lower integration limit over $\epsilon$ is $\epsilon=\rho^{2}$, which guarantees infrared-safety. The upper integration limit is $\epsilon=\rho$, as for the fragmentation function case. The SDG differential cross-section is therefore

$$
\left.\frac{1}{\sigma} \frac{d \sigma}{d \rho}\left(Q^{2}, \rho\right)\right|_{\mathrm{SDG}}=\frac{C_{F}}{2 \beta_{0}} \int_{\rho^{2}}^{\rho} \frac{d \epsilon}{\epsilon} \bar{A}_{\mathrm{eff}}\left(\epsilon Q^{2}\right) \dot{\mathcal{F}}(\rho, \epsilon),
$$

where $\dot{\mathcal{F}}(\rho, \epsilon)$ is given by $(52)$.

Proceeding along the lines of the previous section we first use (37) to write a Borel representation of the SDG cross-section. The Borel function is 23]

$$
B_{\mathrm{SDG}}(u, \rho)=\frac{1}{\rho}\left[\frac{2}{u} \exp \left(2 u \ln \frac{1}{\rho}\right)-\left(\frac{2}{u}+\frac{1}{1-u}+\frac{1}{2-u}\right) \exp \left(u \ln \frac{1}{\rho}\right)\right] .
$$

Next, the exponent in Laplace space [24, 23] is

$$
\ln J_{\nu}\left(Q^{2}\right)=\left.\int_{0}^{1} \frac{1}{\sigma} \frac{d \sigma}{d \rho}\left(Q^{2}, \rho\right)\right|_{\mathrm{SDG}}\left(e^{-\nu \rho}-1\right) d \rho .
$$

The corresponding Borel function is given by

$$
\begin{aligned}
B_{\nu}(u) & =\int_{0}^{1} \frac{d \rho}{\rho}\left[\frac{2}{u} e^{2 u \ln \frac{1}{\rho}}-\left(\frac{2}{u}+\frac{1}{1-u}+\frac{1}{2-u}\right) e^{u \ln \frac{1}{\rho}}\right]\left(e^{-\nu \rho}-1\right) \\
& \simeq \frac{2}{u}\left[e^{2 u \ln \nu} \Gamma(-2 u)+\frac{1}{2 u}\right]-\left(\frac{2}{u}+\frac{1}{1-u}+\frac{1}{2-u}\right)\left[e^{u \ln \nu} \Gamma(-u)+\frac{1}{u}\right],
\end{aligned}
$$

where non-logarithmic terms were neglected. It is interesting to compare this result with the fragmentation function case (40) and (41), where only the collinear limit $\epsilon=1-x=\rho$ plays a role. The terms asociated with the collinear limit are common. Equation (56) contains in addition a term that corresponds to large-angle emission $\epsilon=\rho^{2}$. The latter 
has a double argument $u \longrightarrow 2 u$, indicating larger perturbative coefficients as well as enhanced power-corrections 23].

Equation (56) summarizes the DGE result for the jet-mass distribution. Performing the Borel integral as in (40) and the inverse Laplace transform, this result can be directly used for phenomenological analysis of the thrust and heavy jet mass distribution [23, 44]. A detailed analysis of the perturbative structure of the exponent (sub-leading logs) and of the expected power corrections was performed in 23]. The main results are the following.

Sudakov logs: Performing the Borel integral term by term with the one-loop running coupling we can write the exponent $\ln J_{\nu}\left(Q^{2}\right)$ as in eq. (42) with

$$
f_{k}(\xi)=\sum_{l=0}^{(k-1) / 2} \frac{\left(-\pi^{2}\right)^{l}}{(2 l+1) !}\left[g_{k}^{(l)}(\xi) M_{\mathrm{col}}(k)+g_{k}^{(u)}(2 \xi) M_{\mathrm{la}}(k)\right]
$$

where $\xi \equiv \bar{A}\left(Q^{2}\right) \ln \nu$ and $g_{k}^{(l)}(\xi)$ is given by (44). $M_{\text {col }}(k)$ (given by (45)) and

$$
M_{\mathrm{la}}(k)=-2^{k-1} c_{k-2}
$$

correspond to the collinear and large-angle contributions to the Borel function, respectively. Explicitly, this yields

$$
\begin{aligned}
& f_{1}(\xi)=2(1-\xi) \ln (1-\xi)-(1-2 \xi) \ln (1-2 \xi) \\
& f_{2}(\xi)=-2 \gamma_{E}(\ln (1-\xi)-\ln (1-2 \xi))-\frac{3}{2} \ln (1-\xi)
\end{aligned}
$$

and

$$
\begin{aligned}
& f_{3}(\xi)=0.804 /(1-\xi)-2.32 /(1-2 \xi) \\
& f_{4}(\xi)=0.779 /(1-\xi)^{2}-2.68 /(1-2 \xi)^{2} \\
& f_{5}(\xi)=2.32 /(1-\xi)^{3}-5.00 /(1-2 \xi)^{3} \\
& f_{6}(\xi)=6.12 /(1-\xi)^{4}-15.32 /(1-2 \xi)^{4} \\
& f_{7}(\xi)=23.8 /(1-\xi)^{5}-61.12 /(1-2 \xi)^{5} \\
& f_{8}(\xi)=120.9 /(1-\xi)^{6}-305.52 /(1-2 \xi)^{6} \\
& f_{9}(\xi)=721.54 /(1-\xi)^{7}-1833.55 /(1-2 \xi)^{7} \\
& f_{10}(\xi)=5043.48 /(1-\xi)^{8}-12834 /(1-2 \xi)^{8}
\end{aligned}
$$

The factorially increasing coefficients of $f_{k}(\xi)$ and the enhanced singularity at $\xi=1 / 2$ imply that a fixed logarithmic accuracy calculation holds only in a very restricted region $\xi \ll 1 / 2$. Otherwise, these sub-leading logs must be resummed [23]. Having performed such resummation and having included the appropriate power corrections (see below) the region of applicability of the result can be extended up to $\xi<\xi_{\max }=1 / 2$. We saw that in the absence of large angle soft emission sensitivity (the fragmentation function case) this region is larger: $\xi<\xi_{\max }=1$.

Power corrections: The ambiguity of the Borel integral indicates specific power corrections 23]. Taking into account the singularities of (56) and the factor $\sin \pi u / \pi u$ of eq. (37), the Borel integrand (in the large- $\beta_{0}$ limit) is singular at half integers $u=\frac{1}{2}, \frac{3}{2}, \frac{5}{2} \ldots$, and at $u=1,2$. The first type of singularity is a result of the large angle soft emission 
(the first term in (56)). It translates to power corrections of the form $\sim(\nu / Q)^{n}$ where $n$ is an odd integer,

$$
J_{\nu}^{\mathrm{NP}}\left(Q^{2}\right)=\exp \left\{\sum_{i=1}^{\infty} \frac{2 C_{F}(-1)^{i+1}}{\pi \beta_{0}(2 i-1)^{2}(2 i-1) !} \omega_{(2 i-1) / 2}\left(\frac{\bar{\Lambda} \nu}{Q}\right)^{2 i-1}\right\} .
$$

The large angle power corrections are important when $\rho \sim 1 / \nu$ approaches $\bar{\Lambda} / Q$. They can be resummed into a non-perturbative shape function [17] 23], corresponding to the inverse Laplace transform of (60). Thus, the calculation of the exponent in the large- $\beta_{0}$ limit by DGE yields highly non-trivial information on the shape function: its even central moments (controlling corrections $\sim(\nu / Q)^{n}$ with even $n$ ) are suppressed [23]. The second type of singularity is a result of collinear emission. As in the fragmentation function case, it translates into power corrections of the form $\sim 1 /\left(Q^{2} \rho\right)$ and $\sim 1 /\left(Q^{4} \rho^{2}\right)$. These corrections can be neglected in the region $\rho Q / \gtrsim \bar{\Lambda}$.

\section{Deep inelastic structure functions}

Deep inelastic structure functions differ from the previous examples by the fact that they have a systematic expansion in the powers of the hard scale - the twist expansion [45, 46]. In the region of interest, $x_{\mathrm{Bj}}=Q^{2} /(2 P q) \longrightarrow 1$ (here $P$ is the nucleon momentum and $q$ is the virtual photon momentum $\left.\left(-q^{2} \equiv Q^{2}\right)\right)$ the twist expansion breaks down and power corrections of the form $1 / Q^{2 n}\left(1-x_{\mathrm{Bj}}\right)^{n}$ become dominant. In this respect, the situation is analogous to the fragmentation function case.

The renormalon approach was used to study the $x_{\mathrm{Bj}}$ dependence of power corrections (see [47] [51] and [29]). The correspondence between the renormalon approach and the twist expansion was addressed in [14] in the specific case of the longitudinal structure function $F_{L}$. It was shown there that the infrared renormalon ambiguity of the twist-two coefficient functions cancels against the ambiguity of the twist-four matrix elements, ambiguity that results from the ultraviolet quadratic divergence of these matrix elements. The case of the transverse structure function $F_{2}$ is more interesting than that of $F_{L}$, owing to the threshold region [35]. The matching of the renormalon ambiguity and the twist expansion in this case is discussed in [52].

Contrary to the $e^{+} e^{-}$examples analysed above, in the case of deep inelastic scattering, both incoming and outgoing quarks can be the source of gluon bremsstrahlung. Nevertheless, in the approximation considered (and in the appropriate gauge) one can describe the radiation as if it were associated with the final-state quark alone. Indeed, we will see that the SDG characteristic function, which generates the log-enhanced part of the $F_{2}$ coefficient function, is identical to that of the quark fragmentation function.

Identifying the incoming quark momentum $P$ with $\bar{p}$ ("-" direction) and fixing the axial gauge $A_{+}=0$ we consider gluon emission $(k)$ off the outgoing quark, which carries momentum $p$ in the " + " direction. In this gauge the gluon coupling to the incoming quark does not give rise to singular terms. Momentum conservation implies that $\bar{p}+q=p+k$. The standard $x_{\mathrm{Bj}}$, expressed in terms of the light-cone parameters (6) is

$$
x_{\mathrm{Bj}}=Q^{2} /(2 P q)=Q^{2} /(2 \bar{p} q)=1-(\alpha+\lambda) /(1+\beta) .
$$


The matrix element for emission off the outgoing quark $p$ is (91). The phase-space integral is

$$
\begin{aligned}
& \int \frac{d^{4} k}{(2 \pi)^{3}} \delta\left(k^{2}-m^{2}\right) d^{4} p \delta\left(p^{2}\right) \delta^{4}(\bar{p}+q-k-p) \\
= & \frac{1}{8 \pi^{2}} \int \frac{d \alpha d \beta}{(1+\beta)^{2}} \delta\left(\alpha-\left(1-x_{\mathrm{Bj}}-\epsilon x_{\mathrm{Bj}}\right)(1+\beta)\right),
\end{aligned}
$$

where $\epsilon \equiv k^{2} / Q^{2}=\lambda /\left(x_{\mathrm{Bj}}(1+\beta)\right)$. Expressing (9) in terms of $x_{\mathrm{Bj}}$ and $\beta$, we find that the partonic differential cross-section for a single gluon emission coincides with (25) upon replacing $1-x$ by $1-x_{\mathrm{Bj}}$. As in the $e^{+} e^{-}$case, the integration over $\beta$ is bounded from below by the positivity of the squared transverse momentum. For vanishing transverse momentum $\alpha \beta=\lambda$ one finds

$$
\beta=\frac{\epsilon x_{\mathrm{Bj}}}{1-x_{\mathrm{Bj}}-\epsilon x_{\mathrm{Bj}}} \simeq \frac{\epsilon}{1-x_{\mathrm{Bj}}-\epsilon}
$$

Integrating the gluon emission probability over $\beta$ and substituting the limit (63), one gets the following characteristic function

$$
\left.\dot{\mathcal{F}}(x, \epsilon)\right|_{\log }=\frac{2}{1-x_{\mathrm{Bj}}}-\frac{\epsilon}{\left(1-x_{\mathrm{Bj}}\right)^{2}}-\frac{\epsilon^{2}}{\left(1-x_{\mathrm{Bj}}\right)^{3}},
$$

which is identical to the fragmentation function case (64). This result can be compared with the full characteristic function computed in [47, 29]. The additional terms in the latter do not contribute to log-enhanced terms in the perturbative coefficients. In the approximation considered, the integration over the gluon virtuality has the same range as in the fragmentation function case, i.e. $\epsilon<1-x_{\mathrm{Bj}}$. Also here there is no restriction from below (the coefficient functions are not collinear-safe) so that factorization into a non-perturbative parton distribution must be used. It follows that the structure of the exponent in the large- $\beta_{0}$ limit is just identical to that of the fragmentation function case, as summarized by eqs. (40) and (41). As discussed at the end of section 6 , this structure has definite implications in both the perturbativel and non-perturbative levels. The fact that the exponent is identical to the fragmentation function case suggests that it is the general properties of the quark jet evolution that control the power corrections and not the particular process from which the jet emerges.

\section{Initial-state radiation in the Drell-Yan process}

The classical example where initial-state radiation determines the cross-section near threshold is the case of the Drell-Yan process, the inclusive cross-section for lepton pair production in hard hadronic collisions. Resummation of the leading and next-to-leading logs

"The resummation of deep inelastic coefficient function was recently performed $[53$ to NNLL accuracy. Our results are consistent with those calculations. 
for this process in the limit where $x \equiv Q^{2} /(p+\bar{p})^{2}$ approaches 1 was performed [4, 5, 30] and the associated power corrections were discussed in [25, 17, 26, 27.

The factorization property of soft and collinear gluons holds for initial-state radiation exactly as for final-state radiation. In particular, at the level of the squared matrix element, the emission of soft or collinear gluons appears as an overall factor, multiplying the cross-section for no emission, as in eq. (9). However, as we shall see below, the way the singularity of the propagator is related to the physical parameter that controls the phase space, $1-x$, is fundamentally different and consequently so is the structure of the exponent. Although the leading and next-to-leading logs are similar to those of the jet mass (or the thrust) distribution in $e^{+} e^{-}$annihilation, the characteristic function and thus the sub-leading logs and the power corrections [26] are different.

Consider now the case of quark $(p)$ antiquark $(\bar{p})$ annihilation into an off-shell boson $q$ (a photon in Drell-Yan), where the quarks originate in the colliding hadrons. In the perturbative calculation, we begin with on-shell partons $p^{2}=\bar{p}^{2}=0$. Choosing the frame such that $p$ is in the " + " direction and the gauge $A_{+}=0$, so that the radiation decouples from $\bar{p}$, we start by calculating the squared matrix element for a single gluon emission off the quark $p$. The gluon momentum $k$ is decomposed as in (6). Using (10) and the following phase-space integral

$$
\int \frac{d^{4} k}{(2 \pi)^{3}} \delta\left(k^{2}-m^{2}\right) d^{4} q \delta\left(q^{2}-2 p \bar{p} x\right) \delta^{4}(q+k-p-\bar{p})=\int \frac{d \alpha d \beta}{8 \pi^{2}} \delta(1-x-\beta-\alpha+\lambda),
$$

we find that the partonic differential cross-section for a single gluon emission is

$$
\frac{1}{\sigma} \frac{d \sigma}{d x}=\frac{C_{F} \alpha_{s}}{2 \pi} \int_{\beta_{1}}^{\beta_{2}} d \beta\left[\frac{-\lambda}{(1-x-\beta)^{2}}(1-\beta)+\frac{1}{1-x-\beta} \frac{\beta^{2}-2 \beta+2}{\beta}\right] .
$$

The integration over $\beta$ should now be performed for fixed $m^{2}$ and $s=2 p \bar{p}$, thus for a fixed $\lambda$. As in the previous cases, the relevant integration limit is deduced from the condition that the gluon transverse momentum vanishes, i.e. $\alpha \beta=\lambda$. Substituting the relation $\alpha=1-x-\beta+\lambda$, one obtains a quadratic equation for $\beta$ whose solutions are $\beta_{1,2}=(1-x+\lambda \pm \Delta) / 2$, where $\Delta \equiv \sqrt{(1-x)^{2}-2 \lambda(1+x)+\lambda^{2}}$. Integrating (65) one obtains the following characteristic function:

$$
\left.\mathcal{F}(x, \lambda)\right|_{\log }=\frac{2}{1-x} \ln \frac{(1-x-\lambda+\Delta)(1-x+\lambda+\Delta)}{(1-x-\lambda-\Delta)(1-x+\lambda-\Delta)},
$$

where, as in (28), a factor of $C_{F} \alpha_{s} /(2 \pi)$ was extracted. Since in this case only the small $\beta$ region contributes to log-enhanced terms, the only relevant term in (65) is

$$
\frac{1}{\sigma} \frac{d \sigma}{d x} \simeq \frac{C_{F} \alpha_{s}}{2 \pi} \int_{\beta_{1}}^{\beta_{2}} d \beta\left[\frac{2}{(1-x-\beta) \beta}\right]
$$

This simple expression is sufficient for the calculation of (66).

Taking the logarithmic derivative with respect to $\lambda$ yields [26]

$$
\left.\dot{\mathcal{F}}(x, \lambda)\right|_{\log }=\frac{4}{\sqrt{(1-x)^{2}-2 \lambda(1+x)+\lambda^{2}}} .
$$


Comparing this characteristic function to the case of $e^{+} e^{-}$annihilation (32) or (52) and deep-inelastic scattering (64), it is clear that the structure of the perturbative expansion and the power corrections will be completely different. This should be contrasted with the picture one might get based on the NLL resummation formula: in the so-called deep inelastic factorization scheme [30], the latter is identical to that of the thrust. In fact, the similarity of the leading logs is superficial. There is a fundamental difference between the first set of examples, where the radiation originates in an outgoing quark (an evolving quark jet) with a constrained invariant mass, and the Drell-Yan case, where the radiation originates in the incoming quarks and the total energy $(\alpha+\beta)$ in the final state is restricted. As we shall see this difference is encoded into the structure of the exponent making an impact on both the sub-leading Sudakov logs and the power corrections.

We would like to express the SDG cross-section in the form of a Borel sum, as in (38). We should therefore calculate $\int d \lambda \lambda^{-u-1} \dot{\mathcal{F}}(x, \lambda)$. As in the cases of the fragmentation functions and deep inelastic structure functions, the lower limit is zero so the integral diverges. Factorization must be used to obtain a well-defined Borel function. Using the same subtraction prescription as in (33) we get

$$
\begin{aligned}
B_{\mathrm{SDG}}(x, u)= & \frac{4}{1-x} \int_{1}^{(1-\sqrt{x})^{2}} d \lambda \lambda^{-u-1} \\
& +\int_{0}^{(1-\sqrt{x})^{2}} d \lambda \lambda^{-u-1}\left[\frac{4}{\sqrt{(1-x)^{2}-2 \lambda(1+x)+\lambda^{2}}}-\frac{4}{1-x}\right] .
\end{aligned}
$$

The upper integration limit was deduced from the condition $\beta_{2} \leq \beta_{1}$, yielding $\Delta\left(\lambda_{\max }\right)=0$, and therefore $\lambda_{\max }=(1-\sqrt{x})^{2}$. Since the integration over $\lambda$ is restricted to $\lambda \ll 1-x$, in the logarithmic approximation one can replace the integration limit by $\lambda_{\max } \simeq(1-x)^{2} / 4$ and approximate the $\lambda$-dependent denominator in (69) by $\sqrt{(1-x)^{2}-4 \lambda}$. We thus find

$$
B_{\mathrm{SDG}}(x, u)=\frac{4}{1-x} \frac{1}{u}\left[1-\frac{\sqrt{\pi} \Gamma(1-u)}{\Gamma\left(\frac{1}{2}-u\right)}\left(\frac{1-x}{2}\right)^{-2 u}\right] .
$$

Finally, the Borel function corresponding to the exponent in Mellin space is

$$
B_{N}(u)=\int_{0}^{1} d x\left(x^{N-1}-1\right) B_{\mathrm{SDG}}(x, u)=2\left(e^{2 u \ln N}-1\right) \Gamma(-u)^{2}-\frac{4}{u} \ln N .
$$

As far as the leading terms at large $N$ are concerned, this result agrees with that of ref. [26], which used the full matrix element.

Sudakov logs: Equation (71) allows us to examine the structure of the exponent to a fixed logarithmic accuracy, as in (42). Here $f_{k}(\xi)$ are given by

$$
f_{k}(\xi)=\sum_{l=0}^{(k-1) / 2} \frac{\left(-\pi^{2}\right)^{l}}{(2 l+1) !} g_{k}^{(u)}(2 \xi) M_{\text {in }}(k, l)
$$

where $g_{k}^{(l)}(\xi)$ is defined in (44) and

$$
M_{\text {in }}(k, l)=2 \sum_{j=-1}^{k-2 l-2} c_{k-2 l-3-j} c_{j}
$$


Explicitly, this yields

$$
\begin{aligned}
& f_{1}(\xi)=2(1-2 \xi) \ln (1-2 \xi)+4 \xi \\
& f_{2}(\xi)=-4 \gamma \ln (1-2 \xi)
\end{aligned}
$$

and

$$
\begin{aligned}
& f_{3}(\xi)=1.33 /(1-2 \xi)+6.58 \xi \\
& f_{4}(\xi)=2.12 /(1-2 \xi)^{2} \\
& f_{5}(\xi)=4.00 /(1-2 \xi)^{3}-19.48 \xi \\
& f_{6}(\xi)=11.59 /(1-2 \xi)^{4} \\
& f_{7}(\xi)=48.42 /(1-2 \xi)^{5}+91.56 \xi \\
& f_{8}(\xi)=238.80 /(1-2 \xi)^{6} \\
& f_{9}(\xi)=1438.66 /(1-2 \xi)^{7}-527.14 \xi \\
& f_{10}(\xi)=10078.0 /(1-2 \xi)^{8} .
\end{aligned}
$$

As in the previous examples, sub-leading logs are enhanced by factorially increasing coefficients as well as an increasing singularity at $\xi=\xi_{\max }=1 / 2$.

Power corrections: The power corrections in the $x \longrightarrow 1$ region can be deduced from the singularity of the Borel transform of the exponent (71). As in the previous examples, they are expected to exponentiate together with the perturbative sum. The singularities of the Borel integrand are located at integer values of $u$. Equation (71) has double poles at all integers, but the $\sin (\pi u) / \pi u$ factor of (37) leaves only simple poles. As observed in [26], this singularity structure implies that the leading power correction at large $Q^{2}$ (and not too large $x$ ) is $1 / Q^{2}(1-x)^{2}$. Closer to $x=1$ sub-leading power corrections of the form $1 /\left(Q^{2}(1-x)^{2}\right)^{n}$, where $n$ is an integer, become important. The non-perturbative correction factor (in Mellin space) is

$$
C_{\mathrm{NP}}\left(N, Q^{2}\right)=\exp \left\{\sum_{n=1}^{\infty} \frac{C_{F}(-1)^{n}}{\beta_{0} n(n !)^{2}} \omega_{n}\left(\frac{\bar{\Lambda}^{2} N^{2}}{Q^{2}}\right)^{n}\right\} .
$$

\section{Conclusions}

The factorization of soft and collinear gluon bremsstrahlung is one of the fundamental tools of perturbative QCD [1]. In particular, it sets the basis for resummation of logarithms in many applications. In this paper we demonstrated that factorization holds also in the case of an off-shell gluon. The main advantage in keeping the gluon off-shell is that the virtuality provides the right physical scale for the coupling. This opens the possibility to resum a full set of radiative corrections, which are associated with dressing the emitted gluon (a renormalon sum). Keeping the gluon off-shell, we identified the appropriate kinematic approximation needed for the calculation of log-enhanced terms originating in either the collinear or the soft regions of phase space. The factorization formula led to the definition of a generalized splitting function that describes the emission probability of an off-shell gluon off a quark. As always, factorization results in exponentiation. Thus, DGE promotes the leading order calculation to an all-order sum in two respects: the first 
by dressing the gluon and the second by taking into account multiple emission through exponentiation.

In the class of problems analysed here, where a kinematic threshold imposes a stringent restriction on the real emission phase space, resummation is necessary even for a qualitative description of the differential cross-section. Currently available and future experimental data provide a challenge to QCD: quantitative predictions for differential cross-section are required. The threshold region turns out to be phenomenologically important in many cases: for example in $e^{+} e^{-}$annihilation the cross-section is large only in the two-jet region.

The analysis of the exponent in the different examples considered (see also [23]) shows that sub-leading Sudakov logs are enhanced factorially with respect to the leading logs. This is a direct consequence of the integration over the running coupling, which reveals the presence of infrared renormalons. Thus because of the running coupling, a quantitative description of the threshold region, e.g. in the fragmentation function case $Q^{2}(1-x) \gtrsim \bar{\Lambda}$ or $\left(\beta_{0} \alpha_{s} / \pi\right) L \lesssim 1$ (where $L=\ln 1 /(1-x)$ ), requires the calculation of the exponent to any logarithmic accuracy. Resummation with a fixed logarithmic accuracy, e.g. NLL, applies only for $\left(\beta_{0} \alpha_{s} / \pi\right) L \ll 1$. Moreover, in the threshold region, non-perturbative corrections are particularly significant. As opposed to single-scale observables, non-perturbative corrections are not dominated by a leading power, but rather appear as a convolution of the perturbative distribution with some shape function [17]-[23] of the relevant scale. We saw that the exponent calculated through DGE contains both perturbative and nonperturbative information. As in the case of single-scale observables [13], these two aspects cannot be separated and must be treated together by resummation and parametrization of power corrections.

As any renormalon-based approach, DGE does not pretend to supply a field theoretic definition of the shape function or to determine the magnitude of the non-perturbative corrections. However, it strongly suggests: (1) the factorization of the non-perturbative corrections; (2) the scale on which the non-perturbative function depends, e.g. $(1-x) Q^{2}$ in the fragmentation and deep inelastic cases and $Q(1-T)$ and $Q(1-x)$ in the thrust and Drell-Yan cases, respectively; and (3) the specific powers of this scale appearing in the exponent (in moment space), for example: odd powers of $\nu / Q$ in the thrust, $\left(N / Q^{2}\right)^{n}$, where $n=1$ and 2, in both the fragmentation and deep inelastic cases, and even powers of $N / Q$ in the Drell-Yan case. The first two properties can be deduced also from other considerations. The third is harder to access non-perturbatively. Here one really relies on the assumption that the dominant non-perturbative effects are the ones detected by the perturbative tools.

Acknowledgements: I wish to thank Yuri Dokshitzer, Gregory Korchemsky, Johan Rathsman, Gavin Salam and Douglas Ross for very interesting and helpful discussions.

\section{References}


[1] Y. L. Dokshitzer, V. A. Khoze, A. H. Mueller and S. I. Troian, "Basics of perturbative QCD," (in "Basics of" series, Ed. Frontières, Gif-sur-Yvette, 1991), 274 p.

[2] G. Sterman, Nucl. Phys. B281 (1987) 310.

[3] J. C. Collins, D. E. Soper and G. Sterman, Nucl. Phys. B308 (1988) 833.

[4] S. Catani and L. Trentadue, Nucl. Phys. B327 (1989) 323.

[5] H. Contopanagos, E. Laenen and G. Sterman, Nucl. Phys. B484 (1997) 303 hepph/9604313.

[6] F.E Low, Phys. Rev 110, (1958) 974.

[7] T. H. Burnett and N. M. Kroll, Phys. Rev. Lett. 20 (1968) 86.

[8] V.N. Gribov, "Bremsshtralung of hadrons at high energies", Sov. J. Nucl. Phys., 5 (1967) 280, Yad. Fiz., 5 (1967) 399.

[9] V. Del Duca, Nucl. Phys. B345 (1990) 369.

[10] M. Chaichian and B. Ermolaev, Nucl. Phys. B451 (1995) 194.

[11] S.J. Brodsky, G.P. Lepage and P.B. Mackenzie, Phys. Rev. D28 (1983) 228; G.P. Lepage and P.B. Mackenzie, Phys. Rev. D48 (1993) 2250.

[12] S. J. Brodsky, E. Gardi, G. Grunberg and J. Rathsman, Phys. Rev. D63 (2001) 094017 hep-ph/0002065.

[13] E. Gardi and G. Grunberg, JHEP 9911 (1999) 016 hep-ph/9908458.

[14] M. Beneke, Phys. Rep. 317 (1999) 1 [hep-ph/9807443].

[15] A.H. Mueller, Nucl. Phys. B250 (1985) 327; Phys. Lett. B308 (1993) 355.

[16] V.I. Zakharov, Nucl. Phys. B385 (1992) 452.

[17] G.P. Korchemsky and G. Sterman, Nucl. Phys. B437 (1995) 415.

[18] G.P. Korchemsky and G. Sterman, hep-ph/9505391 30th Rencontres de Moriond, QCD and high energy hadronic interactions, Les Arcs, 1995. ed. J. Tran Thanh Van (Editions Frontières, Gif-sur-Yvette, 1995), p.383.

[19] G. P. Korchemsky, Shape functions and power-corrections to the event shapes, hepph/9806537.

[20] G. P. Korchemsky and G. Sterman, Nucl. Phys. B555 (1999) 335 hep-ph/9902341.

[21] G. P. Korchemsky and S. Tafat, JHEP 0010 (2000) 010 hep-ph/0007005.

[22] A. V. Belitsky, G. P. Korchemsky and G. Sterman, hep-ph/0106308. 
[23] E. Gardi and J. Rathsman, Nucl. Phys. B609 (2001) 123 [hep-ph/0103217.

[24] S. Catani, L. Trentadue, G. Turnock and B. R. Webber, Nucl. Phys. B407 (1993) 3.

[25] H. Contopanagos and G. Sterman, Nucl. Phys. B419 (1994) 77 hep-ph/9310313].

[26] M. Beneke and V. M. Braun, Nucl. Phys. B454 (1995) 253 hep-ph/9506452.

[27] R. Akhoury, M. G. Sotiropoulos and V. I. Zakharov, Phys. Rev. D56 (1997) 377 hep-ph/9702270.

[28] M. Beneke and V. M. Braun, Phys. Lett. B348 (1995) 513 hep-ph/9411229; P. Ball, M. Beneke and V. M. Braun, Nucl. Phys. B452 (1995) 563 hep-ph/9502300.

[29] Yu.L. Dokshitzer, G. Marchesini and B.R. Webber, Nucl. Phys. B469 (1996) 93.

[30] S. Catani, G. Marchesini and B.R. Webber, Nucl. Phys. B349 (1991) 635.

[31] M. Cacciari and S. Catani, hep-ph/0107138.

[32] M. Dasgupta and B. R. Webber, Nucl. Phys. B484 (1997) 247 [hep-ph/9608394].

[33] M. Beneke, V. M. Braun and L. Magnea, Nucl. Phys. B497 (1997) 297 hep$\mathrm{ph} / 9701309$.

[34] G. P. Korchemsky, Mod. Phys. Lett. A4 (1989) 1257.

[35] G. P. Korchemsky and G. Marchesini, Nucl. Phys. B406 (1993) 225 hepph/9210281; Phys. Lett. B313 (1993) 433.

[36] L.S. Brown and L.G. Yaffe, Phys. Rev. D45 (1992) 398; L.S. Brown, L.G. Yaffe and C. Zhai, Phys. Rev. D46 (1992) 4712.

[37] M. Beneke, Nucl. Phys. B405 (1993) 424.

[38] G. Grunberg, Phys. Lett. B304 (1993) 183; see also Quantum Field Theoretic Aspects of High Energy Physics, Kyffhauser, Germany, September 1993.

[39] A. V. Manohar and M. B. Wise, Phys. Lett. B344 (1995) 407 [hep-ph/9406392].

[40] B. R. Webber, Phys. Lett. B339 (1994) 148 [hep-ph/9408222].

[41] Yu.L. Dokshitzer and B.R. Webber, Phys. Lett. B352 (1995) 451 and B404 (1997) 321.

[42] R. Akhoury and V. I. Zakharov, Phys. Lett. B357 (1995) 646 [hep-ph/9504248].

[43] E. Gardi, JHEP 0004 (2000) 030 [hep-ph/0003179].

[44] E. Gardi and J. Rathsman, "Sudakov logs and renormalons in the thrust and the jet-mass distributions", to be published. 
[45] R. K. Ellis, W. Furmanski and R. Petronzio, Nucl. Phys. B207 (1982) 1; Nucl. Phys. B212 (1983) 29.

[46] R. L. Jaffe and M. Soldate, Phys. Rev. D26 (1982) 49.

[47] M. Dasgupta and B. R. Webber, Phys. Lett. B382 (1996) 273 [hep-ph/9604388].

[48] E. Stein, M. Meyer-Hermann, L. Mankiewicz and A. Schafer, Phys. Lett. B376 (1996) 177 hep-ph/9601356].

[49] M. Meyer-Hermann, M. Maul, L. Mankiewicz, E. Stein and A. Schafer, Phys. Lett. B383 (1996) 463 [Erratum-ibid. B393 (1996) 487] hep-ph/9605229].

[50] M. Maul, E. Stein, A. Schafer and L. Mankiewicz, Phys. Lett. B401 (1997) 100 hep-ph/9612300.

[51] R. Akhoury and V. I. Zakharov, hep-ph/9701378.

[52] E. Gardi, G.P. Korchemsky, D.A. Ross and S. Tafat, "Power corrections in deep inelastic scattering at large Bjorken x", to be published.

[53] A. Vogt, Phys. Lett. B497 (2001) 228 hep-ph/0010146. 